\title{
Complex gene expression in the dragline silk producing glands of the Western black widow (Latrodectus hesperus)
}

\author{
Amanda Kelly Lane ${ }^{1}$, Cheryl Y Hayashi ${ }^{2}$, Gregg B Whitworth ${ }^{1}$ and Nadia A Ayoub ${ }^{1 *}$
}

\begin{abstract}
Background: Orb-web and cob-web weaving spiders spin dragline silk fibers that are among the strongest materials known. Draglines are primarily composed of MaSp1 and MaSp2, two spidroins (spider fibrous proteins) expressed in the major ampullate (MA) silk glands. Prior genetic studies of dragline silk have focused mostly on determining the sequence of these spidroins, leaving other genetic aspects of silk synthesis largely uncharacterized.

Results: Here, we used deep sequencing to profile gene expression patterns in the Western black widow, Latrodectus hesperus. We sequenced millions of 3'-anchored "tags" of cDNAs derived either from MA glands or control tissue (cephalothorax) mRNAs, then associated the tags with genes by compiling a reference database from our newly constructed normalized L. hesperus CDNA library and published L. hesperus sequences. We were able to determine transcript abundance and alternative polyadenylation of each of three loci encoding MaSp1. The ratio of MaSp1:MaSp2 transcripts varied between individuals, but on average was similar to the estimated ratio of MaSp1: MaSp2 in dragline fibers. We also identified transcription of TuSp1 in MA glands, another spidroin family member that encodes the primary component of egg-sac silk, synthesized in tubuliform glands. In addition to the spidroin paralogs, we identified 30 genes that are more abundantly represented in MA glands than cephalothoraxes and represent new candidates for involvement in spider silk synthesis.
\end{abstract}

Conclusions: Modulating expression rates of MaSp1 variants as well as MaSp2 and TuSp1 could lead to differences in mechanical properties of dragline fibers. Many of the newly identified candidate genes likely encode secreted proteins, suggesting they could be incorporated into dragline fibers or assist in protein processing and fiber assembly. Our results demonstrate previously unrecognized transcript complexity in spider silk glands.

Keywords: Spidroin, Major ampullate glands, Alternative polyadenylation, MaSp1, Spider, Tag profiling

\section{Background}

Spiders (Araneae) use silk throughout their lives for functions such as prey capture, sperm webs, egg cases, safety draglines, and dispersal [1]. Orb-web weavers and their relatives (Orbiculariae) spin six or more task-specific silk fibers. Each of these task-specific fibers is synthesized in its own set of specialized abdominal glands [1,2]. For example, dragline silk is synthesized in the major ampullate (MA) glands and egg-case silk is synthesized in the tubuliform glands. Each type of silk has a unique suite of impressive mechanical properties [3]. The dragline silk of

\footnotetext{
*Correspondence: ayoubn@wlu.edu

'Department of Biology, Washington and Lee University, 204 W. Washington St., Lexington, VA 24450, USA

Full list of author information is available at the end of the article
}

orb-weavers approaches the tensile strength of steel and is more extensible than rubber or tendon collagen $[4,5]$. This combination of strength and extensibility makes dragline silk incredibly tough. Tubuliform silk, by contrast, is a stiff fiber that can store a large amount of energy [3]. There is interest in capitalizing on the remarkable variation and combination of material properties of spider silks for medical and industrial applications such as tendon replacements, sutures, and as an environmentally friendly alternative to synthetic fibers [6,7].

Spider silks are composed primarily of large structural proteins termed spidroins (spider fibrous proteins) that are encoded by members of a single gene family. Silk types differ from each other in their constituent spidroins [8,9]. For instance, MA glands express MaSp1

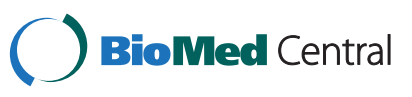


and MaSp2 [10,11] and the outer egg case silk synthesized in tubuliform glands includes TuSp1 [12-14]. Other spidroin paralogs have been identified from each of the gland types except for the aggregate gland [15-18]. Spidroins are large proteins (200-350 kDa, e.g. [19-23]) made up almost entirely of repeated blocks of amino acids (aa), flanked by non-repetitive amino $(\mathrm{N})$ and carboxy $(\mathrm{C})$ terminal domains. These terminal domains are conserved in length $(\sim 150$ aa) and sequence among spidroin types and across species [22,24-26].

The majority of spider silk genetic research has focused on silk synthesized in the MA glands because of the high tensile strength of dragline fibers [4,6,7]. These efforts include characterizing MaSp1 and MaSp2 sequences from multiple species (e.g $[9,22])$ and creating recombinant proteins expressed in transgenic organisms (e.g. $[27,28])$. However, the mechanical properties of fibers spun from recombinant spider silk proteins do not yet accurately mimic natural spider silk fibers (e.g. $[29,30])$, suggesting more extensive work is needed to understand the genetics and spinning of spider silks. Synthesis, assembly, and material properties of dragline silk likely rely on the relative expression of spidroin paralogs as well as the influence of non-spidroin genes. For instance, the relative proportion of $\mathrm{MaSp} 1$ and MaSp2 in dragline silk varies among species and even among individuals of the same species, which could potentially explain inter-and intra-specific level differences in mechanical properties [31-33]. The extensibility of dragline silk correlates positively with its proline content [32,33], an amino acid that is present in higher proportion in MaSp2 than in MaSp1 [11]. The Western black widow, Latrodectus hesperus (Theridiidae), synthesizes draglines with a low proline content and thus MaSp1 is predicted to be approximately three times more abundant than MaSp2 in the fiber [22,34]. The extensibility of black widow dragline silk is correspondingly lower than other species that have draglines primarily composed of MaSp2 [33]. Additionally, MaSp1 is encoded by multiple loci in L. hesperus and the golden orb-weaver Nephila clavipes [35,36]. MaSp1 loci are similar to each other, but not identical, within a species. Variation in the expression patterns of each locus could contribute to variation in mechanical properties of dragline silks within or among species, but such variation in expression has yet to be documented.

Non-spidroin genes can also play important roles in the synthesis and assembly of spider silks, although only a few cases have been described (e.g. [37,38]). For instance, the egg case proteins, ECP-1 and ECP-2, are expressed primarily in the tubuliform glands and are predicted to form cross-links with TuSp1 [37]. Exploring the genetics of spider silk synthesis has been limited to a gene-by-gene approach because of the minimal genomic information available for spiders. Genomic methods have only recently become feasible for non-model organisms, such as spiders, with the advent of next generation sequencing technologies [39-41]. Next generation sequencing and de novo assembly were used to characterize 18,000 "uni-genes" expressed in the silk glands of two spider species, a tarantula and an orb-weaver [42]. This approach identified several major functional classes of genes expressed in silk glands, and discovered new spidroin paralogs, but did not measure relative expression rates. These results represent a major advance in characterizing spider genes, but due to the lack of comparisons with other tissues, it is unclear which genes or functional classes are important for silk synthesis [42].

Here, we use massively parallel signature sequencing (MPSS) to profile expression patterns in MA glands and cephalothoraxes (fused head-body) of the Western black widow. We targeted this species for several reasons. Black widows synthesize dragline silks that are among the strongest measured [5,43]. As descendants of orbweb weaving ancestors [44], black widows possess all the gland types of true orb-web spiders despite building three-dimensional cobwebs [45]. Six of the seven known spidroin paralogs have been described for the Western black widow. Furthermore, because the Western black widow has a relatively small genome for a spider $(\sim 1.3$ billion base pairs [46]), we have arrayed a genomic library and completely sequenced the dragline silk genes MaSp1 and MaSp2 [22] and the prey-wrapping gene AcSp1 [47]. Our aims were to 1) determine relative levels of transcript abundance within MA glands compared to cephalothorax control tissue and 2) identify novel candidate genes of importance to dragline silk production. Our findings demonstrate that dragline silk synthesis involves a much greater transcriptional complexity than previously known, laying the foundation for further studies of silk gland functional genomics and recombinant silk production.

\section{Results and discussion}

Generation of "tags" and construction of reference database We generated more than 20 million tags, which are 3'anchored 20 base cDNA fragments, from mRNA transcripts found in the MA glands and cephalothoraxes of adult female Western black widows using MPSS (Additional file 1: Figure S1; e.g. [48-51]). MPSS allows for highly quantitative comparison between tissues, even in organisms with poorly characterized genomes, by anchoring sequencing to the $3^{\prime}$ end of transcripts. Different transcripts are discriminated by their $3^{\prime}$-most tags and expression levels estimated with higher accuracy than could be achieved by random RNA-Seq [52]. Sequencing more than 5 million tags from each of four libraries (two made from MA glands and two from 
cephalothoraxes) resulted in 200,603 unique tag sequences. Of these, 32,111 unique tags were found at levels greater than one count per million total tags (cpm) in two or more libraries (Table 1 ).

To determine the gene identity of tags, we compiled a non-redundant set of 526 "reference" protein-coding sequences for the Western black widow from 263 newly sequenced cDNAs [GenBank: GW787472-787523; GW820091-800100;JZ531018-531200], 343 published cDNA sequences, and 25 published gene sequences (mitochondrial sequences were excluded from construction of reference database). The only sequences available that included untranscribed regions were for the complete gene sequences of $M a S p 1$ and $M a S p 2$. For initial expression profiling, we thus limited representatives of MaSp1 and MaSp2 to the longest cDNA for each.

The MPSS method we used targeted the $3^{\prime}$-most occurrence of the DpnII recognition sequence, $5{ }^{\prime}$ GATC-3'. We determined tag identity by first predicting all possible tag sequences from the coding or sense strand and the complementary or antisense strand (GATC and 16 following bases) in the reference genes. Predicted tags were then matched to the sequenced tags from our four libraries. Of the 515 sequences in our reference database (when MaSp1 and MaSp2 are represented by one cDNA each), 409 contained a predicted tag and 200 were represented by at least one observed tag.

\section{Identification of differentially expressed transcripts in Western black widows}

An exact test implemented in edgeR [53] identified approximately $23 \%$ of tags were significantly more abundant in one tissue type: 2,323 unique tags were significantly more abundant in the MA glands and 5,010 tags were more abundant in the cephalothoraxes (false discovery rate, FDR $\leq 0.05)$. Each of these differentially expressed tags was at least 1000 times more abundant in one tissue than the other (Additional file 2: Table S1). We assigned 386 unique tags to 200 genes in our reference database (Additional file 1: Figure S2). In terms of abundance and fold change, all tag sequences that matched a reference gene (Figure 1) as well as tags that matched the 3 '-most position (Additional file 1: Figure S3) appear to be an unbiased subset of all observed tags. Abundance levels of these tags were also highly correlated between individual samples (Additional file 1: Figure S4).

Approximately $46 \%$ of the reference genes represented by a sequenced tag were represented by both sense and antisense tags, $45 \%$ were represented by only a sense tag, and $9 \%$ were represented by only an antisense tag (Figure 2 and Additional file 1: Figure S2). Most reference genes were represented by one sense tag and/or one anti-sense tag (Figure 2; average sense tags/gene $=$ 1.325 , range $=0-6 ;$ average antisense tags/gene $=0.82$, range $=0-5$; average total tags/gene $=2.145$, range $=1-9$ ) .

We observed instances of multiple tags aligning to a single gene, but the 3 '-most sense tag was almost always considerably more abundant than other sense tags, as expected (Additional file 1: Figure S5). Genes represented by multiple sense tags were usually represented by multiple antisense tags, but antisense tags did not show a consistent relationship between position on the gene and tag abundance (Additional file 1: Figure S5). Alternative tags could result from incomplete digestion with DpnII, or from degradation of mRNAs. However, in many cases multiple tags represent genuine alternative transcripts (e.g. alternatively spliced or polyadenylated forms). An example of these alternative transcripts is described for MaSp1 below. Because the origin of multiple tags per gene is not always clear, we estimated transcript abundance two ways. First, under the assumption that all tags that matched a gene represent transcription of some kind, we summed the average cpm of all tags from the sense strand, or separately, from the antisense strand (Additional file 1: Figure S6a). Second, to account for the possibility that multiple tags represent alternative transcripts, we estimated transcript abundance based solely on the 3 '-most position of the sense strand, or its matched position on the antisense strand (Additional file 1: Figure S6b). These two estimates are tightly correlated (Additional file 1: Figure S7).

Because we selected for polyadenylated mRNA, we did not expect to capture antisense transcripts. Total abundance of a transcript estimated from summing all tags that matched that gene sequence is correlated between sense and antisense strands, with total abundance of sense tags almost always higher than total abundance of

Table 1 Counts of tags generated from two major ampullate (MA) gland and two cephalothorax CDNA libraries

\begin{tabular}{llll}
\hline & Tags sequenced $>\mathbf{1}$ time & Tags with $>\mathbf{1} \mathbf{~ c p m}^{\mathbf{a}}$ in at least $\mathbf{2}$ libraries & Normalization factor \\
\hline MA silk gland 1 & $6,626,230$ & $6,392,914$ & 1.01 \\
MA silk gland 2 & $6,731,324$ & $6,346,320$ & 1.08 \\
Cephalothorax 1 & $6,587,276$ & $6,316,307$ & 0.87 \\
Cephalothorax 2 & $5,912,202$ & $5,648,579$ & 1.05 \\
Total unique tags & 200,603 & 32,111 & \\
\hline
\end{tabular}

${ }^{\mathrm{a}} \mathrm{cpm}=$ counts per million. 


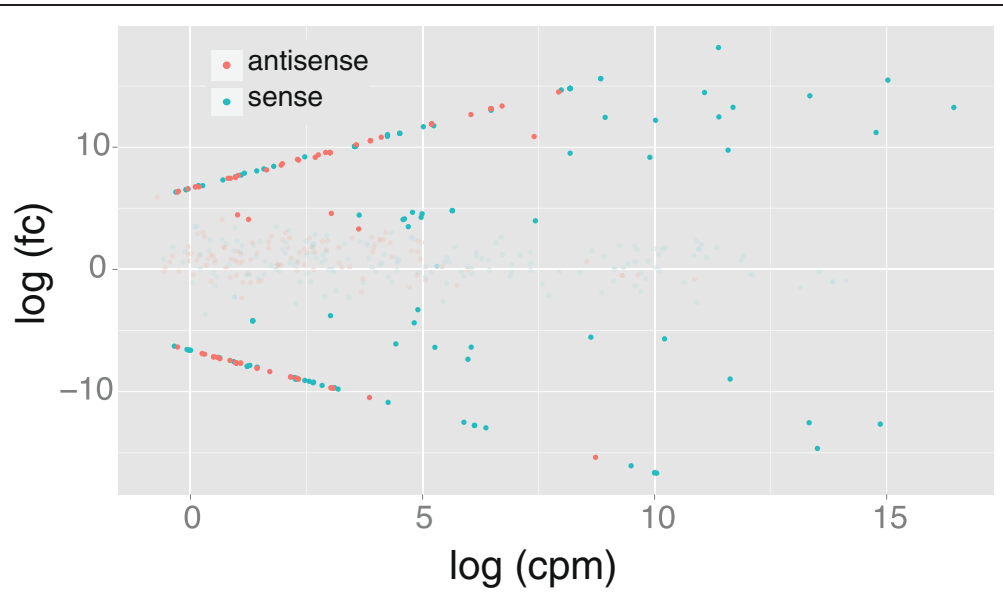

Figure 1 Tag abundance versus log fold change, log (fc), between major ampullate (MA) glands and cephalothoraxes. Tags included are only those that matched a reference gene. Tag abundance, log (cpm), is the natural log of average counts of that tag per million total tags (cpm) among all four libraries. Tags that are differentially expressed between MA glands and cephalothoraxes (FDR $\leq 0.05)$ are bolded. Turquoise tags matched the sense strand and salmon tags matched the antisense strand. A positive log (fc) indicates higher abundance in MA glands.

antisense tags (Additional file 1: Figure S6a). For instance, expression levels based on sense tags varied from 0 to $164,899 \mathrm{cpm}$ in MA glands while antisense counts varied from 0 to $1,287 \mathrm{cpm}$. Despite lower abundance of antisense tags, differences in gene expression levels between tissues estimated by sense and antisense tags are tightly correlated (Additional file 1: Figure S8). Thus, even if our antisense tags represent an experimental error associated with the reverse transcription phase of cDNA synthesis [54], they provide consistent information regarding differential expression.

It is also possible that antisense transcription is widespread in spiders. In metazoan genomes investigated thus far, $5-22 \%$ of genes are represented by both sense and antisense transcripts [55]. In mice and humans, more than $40 \%$ of the transcript pool could result from antisense transcription [54]. Furthermore, expression patterns of sense and antisense transcripts generated from the same genomic locus tend to be correlated [56], similar to the pattern seen here (Additional file 1: Figure S8).

\section{Variable expression of spidroin paralogs in MA glands}

Our database contained 11 unique sequences encoding MaSp1, including one complete protein-coding sequence for locus 1 and several thousand base pairs of non-coding flanking sequence [22]. It additionally included representatives of two other loci (loci 2 and 3) and partial length cDNAs. From these sequences, including non-coding regions, we predicted 123 sense tags (66 unique) and 122 antisense tags (59 unique). Full-length $M a S p 1$ has 11 predicted sense tags ( 8 unique) and 11 predicted antisense tags (7 unique) that matched an observed tag. Importantly, all of the tags predicted in the coding region of the full-length gene were observed as well as two downstream

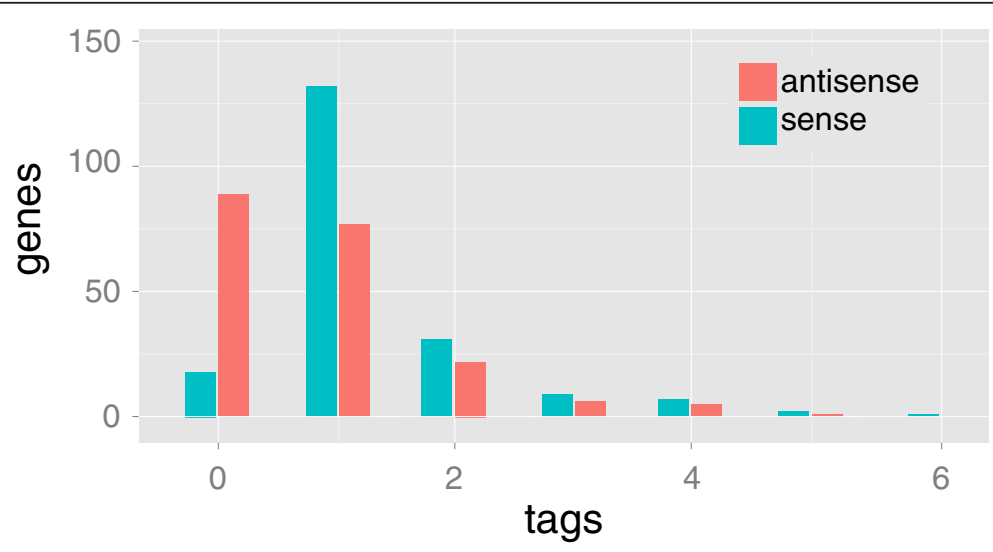

Figure 2 Numbers of black widow reference genes represented by a given number of tags. Tags that matched the sense strand are shown in turquoise and tags that matched the antisense strand are shown in salmon. 
of the coding region (Figure 3). As expected, there were no observed tags that corresponded to intergenic genomic DNA. In addition to the eight unique sense tags that matched the full-length MaSp1 gene, there were five other unique sense tags that aligned to alternative MaSp1 sequences (Figure 3).

Alignment of all MaSp1 sequences indicated that the three loci and the cDNAs share an identical observed sense tag in the C-terminal encoding region (Index 0 , Figure 3). This tag is the 3 '-most predicted one in the cDNAs and was the most abundant observed MaSp1 tag and the second most abundant observed tag in MA glands overall (mean cpm in MA glands $=62,969$; mean $\mathrm{cpm}$ in cephalothoraxes $=1.5$; Figure 3 ). The seven sense tags found throughout the repetitive encoding region of the full-length MaSp1 were much less abundant (mean $\mathrm{cpm}$ in MA glands =14-567), consistent with selecting for the 3 '-most occurrence of the DpnII recognition sequence.

Although the $3^{\prime}$-most tag from the coding sequence cannot discriminate expression levels of each MaSp1 locus, there are other indices that are diagnostic for particular loci (Figure 3). Prior to this study, only locus 2 was supported by expression data [35]. Here, we observed unique tags for all three loci. Locus 2 has an observed unique tag 482 bases downstream of the stop codon (Index 3, Figure 3; mean $\mathrm{cpm}$ in MA glands = 6086). Loci 1 and 3 share an observed tag 144 bases downstream of the stop codon that is not found in locus 2 (Index 1, Figure 3; mean cpm in MA glands =1063). Yet another observed unique tag occurs in locus 1, 486 bases downstream of the stop codon (Index 2, Figure 3; mean $\mathrm{cpm}$ in MA glands=449). A different tag was predicted in the same location in locus 3, but was not observed. However, locus 3 has a unique observed tag in the N-terminal coding region (Index $=-9$, Figure 3; mean cpm in MA glands =10). Thus, we have evidence for the co-expression all three loci at the same time in the same tissue.

The observed tags found downstream of the MaSp1 stop codon also suggest that both loci 1 and 2, and possibly 3 , are alternatively polyadenylated. Consistent with this hypothesis is the occurrence of a polyadenylation signal (AATAAA) downstream of an observed tag in each of these loci (Figure 3). Both 3' UTR length, and specific sequence elements contained therein, have been shown to be important for a variety of regulatory events including control of mRNA stability and translational efficiency [57]. Our results thus raise the possibility that differential polyadenylation of $M a S p 1$ transcripts may be involved in locus-specific stability or translational control.

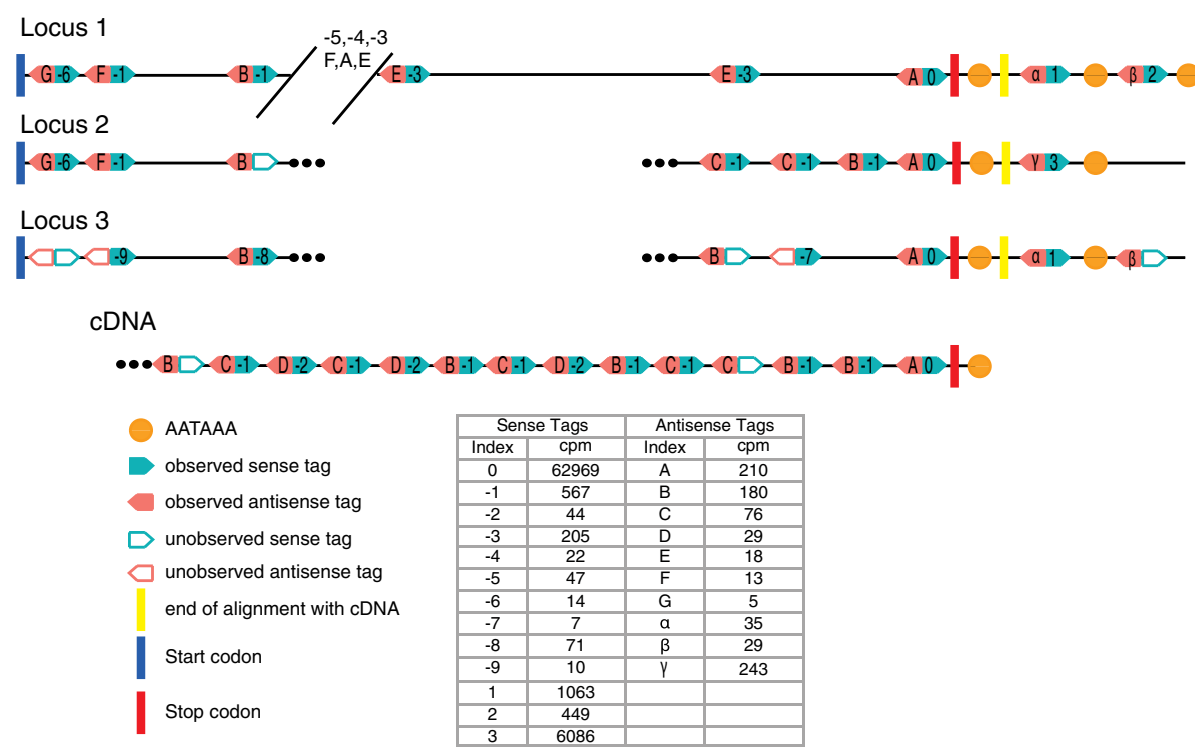

Figure 3 Diagram of all predicted tags for three MaSp1 loci and the longest MaSp1 cDNA. Observed (filled arrows) and unobserved (empty arrows) tag locations are approximate and not to scale. Antisense (salmon) and sense (turquoise) tags are paired by location. These MaSp1 tags are not predicted for any other gene in the reference database. Dots (...) indicate missing sequence data. Slashed lines in Locus 1 indicate $\sim 5$ kilobases of sequence data that is not shown. Observed unique tag indexes are numbered for sense tags $\left(0\right.$ being $3^{\prime}$-most tag in the protein coding sequence and -9 being $5^{\prime}$ most) and lettered for antisense tags (A being the $3^{\prime}$-most of the protein coding sequence and $G$ being the $5^{\prime}$-most). Observed tags that appear after the stop codon are labeled with positive integers (sense indices 1, 2, 3) and Greek letters (antisense indices $\alpha, \beta, \gamma$ ). Tag abundance is the average counts of that tag per million total tags (cpm) in the two major ampullate libraries. Multiple occurrences of some tag sequences reflect the repetitive nature of MaSp1. Start codon (blue hatch), stop codon (red hatch), and the polyadenylation signal AATAAA (orange dot) are indicated. The CDNA aligns with the three genomic loci up to the yellow hatch. 
Although the $3^{\prime}$-most coding region tag of MaSp1 is identical among all three loci (Index 0, Figure 3), the sequences of downstream tags are unique and can therefore be used to compare locus-specific expression levels. Analysis of these tags reveals that the isoform of locus 2 carrying an elongated 3' UTR is $\sim 4$ times more abundant than the alternatively polyadenylated forms of loci 1 and 3 combined (6086 cpm vs. 1512 cpm, respectively; Figure 3). In the absence of strong, locus-specific alternative $3^{\prime}$ end regulation, this finding suggests that locus 2 is dominant in the MA gland tissue of adult females. Our data are therefore consistent with the previously suggested hypothesis that MaSp1 locus variants could be incorporated in differing proportions into draglines [35]. Because MaSp1 encoded by each locus differs in proportion and ordering of ensemble repeats, varying the proportion of each could affect silk properties. Importantly, prior to this study there was no direct evidence for simultaneous expression from all three loci.

Our database also contained seven sequences encoding MaSp2, including a complete gene with several thousand base pairs of non-coding flanking sequence [22]. From these MaSp2 sequences, 184 tag locations (64 from the MaSp2 coding region) were predicted from the sense strand representing 100 unique tag sequences (29 from the MaSp2 coding region). In the full-length gene, 33 tag locations were observed (11 unique tag sequences). Four of these observations were well outside the coding region and appeared at low frequencies in all tissues $(\mathrm{cpm}<6.3)$, leaving seven unique observed sense tags within the MaSp2 coding region (Additional file 1: Figure S9). Alignment of the complete MaSp2 gene with other MaSp2 sequences including partial cDNAs showed that all sequences shared a 3 '-most predicted sense tag. This tag was the most abundant in MaSp2 sequences (mean cpm in MA glands $=18,386$ ). All other observed MaSp2 tags were much less abundant (mean cpm in MA glands=2-68). There was only one additional unique sense tag observed from other MaSp2 sequences. This tag is in the N-terminal encoding region of a partial MaSp2 cDNA (mean cpm in MA glands $=4.39$ ). A tag that only differed by one base pair from this tag was predicted from the corresponding coding region in the full-length gene, but was not observed (Additional file 1: Figure S9).

The ratio of MaSp1:MaSp2 transcript abundance based on the $3^{\prime}$-most coding tags varied between the two individuals, but the ratio based on the average cpm in MA glands is 3:1 MaSp1:MaSp2 consistent with the amino acid composition of MA fibers collected from Western black widows [22,34]. Thus, despite the potential for complex locus-specific translational control, transcript abundance may accurately reflect overall protein abundance of MaSp1 and MaSp2.
Variation in proportion of MaSp1 and MaSp2 could explain variation in fiber properties among individual black widow spiders [58]. In three other species, varying the amount of protein in the spider's diet significantly alters mechanical properties of MA fibers [59]. Specifically, extensibility of MA fibers, a property highly correlated with the proline content of the fiber [32,33], decreased with a low protein or protein-less diet [59]. Since proline is in higher proportion in MaSp2 than in MaSp1 [11], an increased extensibility in MA fibers is likely due to an increase in the proportion of MaSp2 in the fiber. Individual spiders could have widely varying diets that overtime could change the proportion of spidroins in the silk fibers and thus create fibers with varying mechanical properties.

TuSp1, which encodes the primary component of outer egg-cases, was unexpectedly expressed in MA glands. Our non-redundant database contained two sequences predicted to encode TuSp1, but both shared an identical observed tag in the C-terminal encoding region (mean cpm in $\mathrm{MA}=996$; mean cpm in cephalothorax $=0$ ). TuSp1 was surprisingly abundant, ranking $10^{\text {th }}$ most represented gene of the ones that are more abundant in MA glands than cephalothoraxes, directly following MaSp2 (Figure 4). However, one individual transcribed much more TuSp1 (1990 cpm) than the other $(2 \mathrm{cpm})$. The difference in expression levels between the two individuals could be explained by individual differences in readiness to make egg-cases. The number of transcripts encoding TuSp1 and other egg-case proteins increase in tubuliform silk glands as the individual nears egg-case production [60]. The individual with lower levels of TuSp1 in MA glands did not have well-developed eggs. However, the individual with high levels of TuSp1 had large, mature eggs, suggesting that TuSp1 transcription increases in MA glands at the same time as increasing in tubuliform glands. It is possible TuSp1 becomes incorporated in MA fibers at the time of egg-case production, which could contribute to variation in mechanical properties. Alternatively, translational control of TuSp1 prevents protein synthesis in MA glands despite the presence of TuSp1 transcripts. We also cannot exclude the possibility that our detection of TuSp1 in MA glands is due to leakage from mRNAs transcribed in the tubuliform glands.

\section{Novel candidate genes involved in silk synthesis}

In addition to spidroin encoding genes, we identified 30 (28 when analysis is limited to the $3^{\prime}$-most tags) other genes represented by tags that were significantly more abundant in MA glands than cephalothoraxes (FDR $\leq$ 0.05; Figure 4). When translated, 20 (18 when analysis is limited to the 3 '-most tags) of these genes aligned to functionally annotated proteins or conserved domains (Figure 4). Of the remaining ten genes, one did not match 


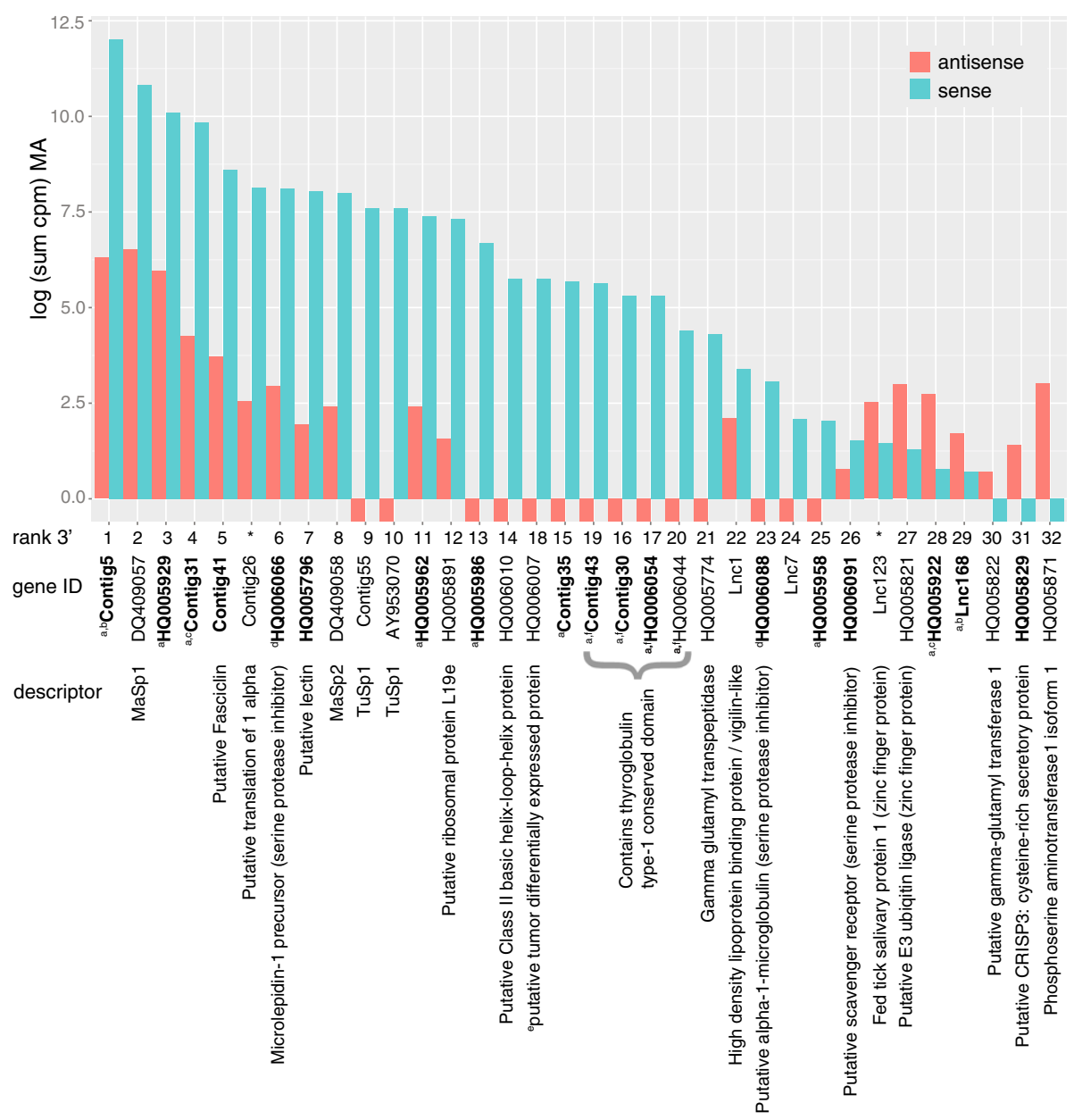

Figure 4 Expression levels of black widow reference genes. Total sense (turquoise) and antisense (salmon) transcript abundance for genes with an observed tag more abundant in major ampullate (MA) glands than cephalothoraxes (FDR $\leq 0.05)$. Transcript abundance is the sum of the average log counts per million (cpm) of all tags that matched to that gene between two MA libraries. Reference genes are ranked according to decreasing total abundance. Rank abundance according to just the $3^{\prime}$-most tag (rank 3') is almost identical (see also Additional file 1: Figure S6c). Genes that were not significantly different according to the $3^{\prime}$-most tag are marked with an asterisk $\left(^{*}\right)$. Descriptor is the top BLASTX hit to NCBI non-redundant (nr) protein database. Bolded genes have a signal peptide predicted by SignalP using D-value cut off = "sensitive". "Only BLASTX hits are hypothetical proteins in L. hesperus. ${ }^{b}$ Contig5 and Lnc168 are nearly identical for the first 178 bases. Tags are located downstream in regions that do not align between the two sequences. ${ }^{c}$ Contig31 and HQ005922 align with $42 \%$ identity over 43 amino acids. ${ }^{\mathrm{d}} \mathrm{HQ} 006066$ and HQ006098 align with 47\% identity over 54 amino acids. ${ }^{e}$ Aligns to hypothetical proteins from multiple species using BLASTX. ${ }^{\text {T These four }}$ sequences align with $~ 73-95 \%$ pairwise identity over the entire amino acid sequence (81-93 amino acids). Contig43, Contig30 and HQ006054 share a tag. Contig43 and HQ006044 share an additional tag. HQ006044 has a predicted tag in the same location as the other three, but it is not observed.

any entries in the NCBI nr database and nine aligned only to hypothetical proteins previously predicted for Western black widows. All but one of these ten encode proteins with signal peptides predicted by SignalP [61] (Figure 4), suggesting they are secreted. Secreted proteins could be components of MA fibers or involved with fiber assembly.

An unanticipated finding was that the $3^{\prime}$-most sense transcript tag of one of the hypothetical proteinencoding genes is three times more abundant in MA glands than that of MaSp1 (Figure 4). This gene, "Contig5", has a short (45 aa) open reading frame (ORF) and SignalP predicts an even smaller mature peptide (19 aa).
The first 178 bases, which includes the first 27 aa, of "Contig5" is nearly identical to that of another gene overrepresented in MA glands, "Lnc168", as well as one ("Lnc106") that was not differentially expressed between MA and cephalothorax. Each of these sequences differs in the last few amino acids and most of the putative 3' UTRs, including the tag locations. "Lnc168" is much less abundant in MA glands than "Contig5" (Figure 4). These sequences may represent transcripts from paralogous genes, alternatively spliced genes, or a combination of both scenarios. Alignment revealed two regions of almost exact identity among all three sequences, and 
another region of exact identity between two, interspersed with unalignable regions. These patterns are consistent with both hypotheses of paralogous genes and alternatively spliced genes. Alternative splicing is an important mechanism for generating functionally distinct proteins with tissue specific expression [62] as well as unique 3' UTRs. Isoforms caused by 3' UTR alternative splicing can modify the production of the protein through altering locations for microRNA binding and activating decay pathways such as the nonsensemediated decay pathway $[63,64]$.

Among the most highly expressed genes in MA glands that could be annotated were ones predicted to encode fasciclin, translation elongation factor 1-alpha, and lectin. Each of these was more abundant than MaSp2 (Figure 4). Abundance ranking of genes expressed in MA glands was almost identical if estimates were based solely on the 3'-most tags (Figure 4; Additional file 1: Figure S6c). Tags corresponding to elongation factor 1alpha were only slightly more abundant in MA glands than cephalothoraxes (log fold change $=1-3.5$ ). In fact, the 3 '-most sense tag of this gene was not significantly different between the two tissues. Thus, the higher abundance in MA glands probably reflects the very high rates of translation to produce dragline spidroins, MaSp1 and MaSp2. In contrast, tags representing fasciclin and lectin-encoding genes were consistently highly abundant in MA glands but either absent or extremely low in cephalothoraxes (log fold change $=9.7-14.5$ ). In various insect species, fasciclins are cell adhesion glycoproteins that play a role in neuronal development (e.g. [65]). Lectins are sugar-binding proteins involved in the innate immune response in Drosophila [66,67]. However, it is not immediately clear how these particular functions might be involved with silk synthesis. It is possible that these genes are expressed in specialized cells within the MA glands that are not secreting silk proteins. The MA glands are divided into multiple regions including a tail and ampullate region where spidroins are expressed, the lower ampullate region where spidroins are stored, and the duct where spidroins begin assembly for ultimate extrusion as fibers $[20,68]$. There may well be cells within any of these regions that have currently unknown functions not found in the cephalothorax.

Thirteen of the 20 genes overrepresented in MA glands that were annotated by sequence similarity are predicted to encode proteins that can be grouped into three functional classes (Figure 4). These include protease inhibitors (three genes align to serine protease inhibitors and four align to cysteine protease inhibitors), proteins with transferase activity (two genes align to gamma-glutamyltransferases and one to phosphoserine aminotransferase), and transcription factors (three genes, or two if analysis is restricted to $3^{\prime}$-most tags, align to conserved DNA binding domains found in transcription factors and thus could serve to increase transcription of spidroins and other silk gland specific proteins). The protease inhibitors appear to be derived from two large gene families. The putative serine protease inhibitor genes encode 54 aa domains that align to each other with $47 \%$ pairwise identity. They also align to four additional translated genes in our reference database that were not overrepresented in MA glands. Similarly, four of the genes overrepresented in MA glands encode thyroglobulin type-1 conserved domains, which function as cysteine protease inhibitors. These four genes align with each other with $85-89 \%$ pairwise nucleotide identity over their entirety. At the amino acid level, they align to an additional four sequences in our reference database. The numerous protease inhibitors expressed in MA glands likely reflect the importance of maintaining protein integrity in the storage compartment of the MA glands. Widow spiders regularly spin dragline silk and need a ready supply of functional spidroins to incorporate into fibers. These upregulated gene family members may be specialized for maintaining spidroins.

One of the genes predicted to encode a protein with transferase activity is homologous to a phosphoserine aminotransferase, which is part of the cascade for serine biosynthesis as shown in Escherichia coli [69]. Another two transcripts encode gamma-glutamyltransferases also known as gamma-glutamyltranspeptidases ( $\gamma$-GT), which in mammals, are primarily utilized in the glutathione pathway [70]. This pathway is implicated in protection against oxidative stress and redox regulation of gene expression [70]. Because spider MA silk processing involves a series of $\mathrm{pH}$ changes in the gland [68], oxidation regulation is likely to be crucial and could be mediated by $\gamma$-GT-like proteins.

\section{Conclusions}

Through cDNA tag profiling, we describe previously unrecognized gene expression complexity in MA glands of the Western black widow. As expected, MaSp1 and MaSp2 were among the most highly expressed genes in MA glands. Surprisingly, the most abundant transcript in MA glands was one with unknown function. Also unexpected, was the discovery of high levels of TuSp1 expression and an additional six genes that were more abundantly represented in MA glands than MaSp2 (Figure 4). In addition, we demonstrated the simultaneous, but unequal expression of three MaSp1 loci. Alternative polyadenlyation of MaSp1 and alternative splicing of other genes upregulated in MA glands may also increase the complexity of individual variation of MA proteins. We propose that modulating the composition of MaSp1 variants, MaSp2, and possibly TuSp1 within silk glands will alter the material properties of dragline 
silk. Hence, if the ratios of these spidroins change as a consequence of ontogeny, physiology, or the environment, this can contribute to variation in the properties of silks spun by the same spider (intraindividual) or different (intraspecific) spiders.

We identified 34 unique gene sequences represented by tags that were significantly more abundant in MA glands than cephalothoraxes. Besides spidroin genes, we found 30 new candidate genes for dragline silk synthesis. Approximately $1 / 3$ of these genes have no known homolog outside of Latrodectus. It is possible that these genes resulted from black widow specific evolutionary events. However, due to the paucity of genomic resources for spiders we cannot exclude the possibility that these genes have homologs in other spiders. Identifying homologs expressed in silk glands of other spider species would strengthen the argument that these genes are involved in silk synthesis. Of the genes predicted to encode proteins with functional homologs some may simply reflect the high rates of translation and protein storage in silk glands such as translation factors, amino transferases, and protease inhibitors. Others are likely transcription factors important for regulating gland-specific spidroin expression. Gamma-glutamyl-transferases may be important regulators of $\mathrm{pH}$ changes in the MA gland that are necessary for fiber assembly. An overriding theme for many of the genes overrepresented in MA glands is that they are members of larger gene families. Neofunctionalization of gene copies expressed in silk glands may be even more important for silk synthesis than the previously recognized diversity of the spidroin gene family. As such, silk glands represent a model system for understanding the evolution of tissue specific functions. Furthermore, our increased understanding of gene regulation in spider silk glands will ultimately lead to improved recombinant silk production.

\section{Methods}

Construction and sequencing of "tag" libraries

Major ampullate (MA) glands and cephalothoraxes were dissected from two Latrodectus hesperus adult females caught live in Riverside, California (USA). Total RNA was isolated from each tissue type for both individuals (four separate isolations) by homogenizing tissue in TRI$\mathrm{zol}^{\odot}$ (Invitrogen, Carlsbad, CA) and further purifying RNA with the RNeasy Mini Kit (Qiagen, Valencia, CA). Any remaining genomic DNA was removed by incubating RNA with DNase from the Ambion ${ }^{\circledR}$ TURBO DNAfree $^{\text {TM }}$ kit (Invitrogen).

We then constructed four cDNA "tag" libraries using the DGE: tag profiling for DpnII kit (Illumina, Inc., San Diego, CA). In brief, we extracted single stranded poly (A) mRNA using Sera-mag magnetic oligo(dT) beads. Single stranded DNA complementary to the mRNA
(cDNA) was synthesized using SuperScript ${ }^{\oplus}$ III Reverse Transcriptase (Invitrogen), primed from the oligo(dT) magnetic beads. Still attached to the magnetic beads, second strand cDNA was synthesized by incubation with RNaseH and DNA Polymerase I. The double stranded cDNA was digested with DpnII, which recognizes $5^{\prime}$ GATC-3'. The digested cDNA was washed and only the portion of cDNA still attached to the magnetic bead was retained. An adapter was ligated to the site of DpnII digestion, introducing the recognition sequence for MmeI. The adapter-cDNA ligation was digested with $\mathrm{MmeI}$, which cuts $20 \mathrm{bp}$ downstream from the recognition sequence and 16 bp downstream of the DpnII site, GATC. A second adapter was then ligated to the MmeI cut site, and the adapter-ligated cDNA construct was enriched by PCR and purified (Additional file 1: Figure S1). The resulting libraries were each sequenced in a separate lane of a flow cell on the Genome Analyzer II DNA Sequencer (Illumina) at the University of California Riverside Genomics Core Instrumentation Facility.

\section{Generation of reference database}

In order to describe novel protein-coding genes from Western black widows, we constructed a normalized cDNA library. Total RNA was extracted and pooled from three adult females by homogenization in TRIzol $^{\circledR}$ (Invitrogen) followed by purification with the RNeasy Mini Kit (Qiagen). mRNA isolation, cDNA synthesis, normalization, library construction, and arraying were performed by BIO S\&T (Montreal, Canada). In brief, cDNA was synthesized from $5 \mu \mathrm{g}$ of mRNA using the SMART $^{\mathrm{TM}}$ cDNA library construction kit (Clontech, USA). Abundant cDNAs were reduced in frequency by subtractive hybridization. This method hybridizes a sample of biotyntilated single stranded cDNA to excess single stranded cDNA copies in the library. Double stranded cDNA is then removed using streptavidin beads. This allows for more efficient library sequencing. Following normalization, cDNAs were amplified, subjected to SfiI digestion, and size fractionated in a $1 \%$ agarose gel. Fragments greater than 0.5 kilobases were purified for cloning. cDNA fragments were ligated into a modified pBluescript II SK(-) vector between EcoRI (5') and XhoI (3') and used to transform bacterial cells (Escherichia coli strain DH10B from Invitrogen). Over 18,000 recombinant clones were arrayed into 48 384well plates. Clones were sequenced using M13R or T3 to obtain the 5 ' sequence of cDNAs (62 previously submitted to NCBI's EST database and 201 newly submitted for this study; Additional file 3: Table S2). Two clones were sequenced with an oligo(dT)-V primer to obtain sequence from the $3^{\prime}$ end of cDNAs.

All 368 published $L$. hesperus nuclear protein-coding sequences were downloaded from the NCBI nucleotide 
database in June 2012 and combined with the ESTs. We generated a non-redundant set of sequences using CAP3 [71], which resulted in 50 contiguous sequences (contigs) and 476 singletons. These 526 sequences represented our reference database for assigning "tags" to genes. We identified homologs of these sequences using BLASTX [72] (universal genetic code, default parameters, e-value cut off $10^{-6}$ ) comparisons to NCBI's non-redundant protein database $(\mathrm{nr})$. We predicted signal peptides from translated genes using SignalP 4.1 [61], with D-cutoff value set to "sensitive". We input the longest ORF starting with a methionine unless BLAST predicted an alternative ORF.

\section{Sequence processing and identification of differentially expressed "tags"}

Illumina sequence reads were converted to fastq files including the base call and quality scores. Adapter and low quality sequences were removed with utilities in Bioconductor's [73] Biostrings package, version 2.24.1 [74] (Additional file 1). Unique 16 base "tags" were identified and counted within each library. Any tags sequenced only once were removed. Tag counts were proxies for the expression level of the associated genes.

We analyzed tag counts from the four libraries with edgeR [53]. We filtered out low abundance tags retaining only tags with a count per million (cpm) of greater than one in at least two of the four libraries [50]. We generated multi-dimensional scaling plots of the libraries using plotMDS [75] and applied TMM (trimmed mean of $\mathrm{M}$ ) normalization to account for the differences in library sizes and composition [76]. The exact test for the negative binomial distribution was used to compare the tag counts from the cephalothorax to the MA libraries [77]. The common dispersion and tagwise dispersion across all libraries were estimated $[77,78]$. The common dispersion is the squared coefficient of variation which gives the amount of variability in the abundance of each tag between replicate libraries, whereas the tagwise dispersion measures this variation for each individual tag rather than the total pool. The false discovery rate (FDR) was estimated using the Benjamini and Hochberg [79] algorithm. We considered genes represented by a tag with an FDR $\leq 0.05$ to be differentially expressed between MA glands and cephalothoraxes.

\section{Matching observed tags to genes in reference database}

To match our observed tags to genes in the reference database, we determined the locations and sequences of possible tags from our reference genes in both the sense and antisense directions. We ensured that all input sequences represented the coding strand (or sense transcript). First, we assumed that all sequences from NCBI's nucleotide database had been correctly annotated. Second, our normalized cDNAs were positionally cloned so that sequencing with M13R or T3 resulted in characterizing the $5^{\prime}$ end of coding sequences. Clones sequenced from the $3^{\prime}$ end of the cDNA were reverse complemented prior to searching for tag sequences. Third, we inspected contigs assembled by CAP3 for retention of directionality. If a contig was reverse complemented relative to the raw sequences, we reverse complemented the contig. Finally, we checked that the coding frames predicted by BLASTX were all positive.

We batch imported our FASTA file of reference gene sequences into an $\mathrm{R}$ data frame and identified the tag positions by searching for "GATC" (Additional file 1). The position information generated above was used to retrieve the 16 bases following the "GATC" to generate the predicted tags from the sense strand. Similarly, 16 bases preceding the "GATC" were retrieved and reverse complemented to generate the predicted tags from the antisense strand. We then merged observed tag counts and statistics from the edgeR analysis with the table of predicted tags to obtain expression information for genes in our reference database.

\section{Relative expression levels of transcripts}

We estimated expression levels of transcripts represented by genes in our database by summing counts for all unique tags that matched that gene sequence. Tags that aligned to more than one location in a sequence were only counted once. Because MaSp1 and MaSp2 were each represented by multiple non-redundant gene and cDNA sequences, we restricted analysis of expression levels to the longest cDNA associated with each gene [GenBank:DQ409057 and GenBank:DQ409058 respectively]. We also estimated expression levels from only the counts of the 3'-most tag of the sense strand, or the matched position for antisense abundance.

\section{Additional files}

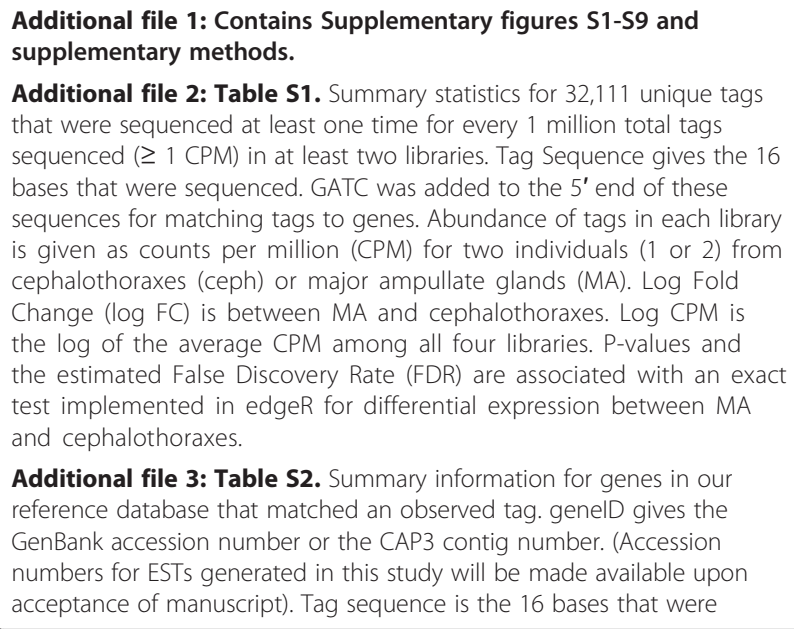

Additional file 3: Table S2. Summary information for genes in our reference database that matched an observed tag. genelD gives the GenBank accession number or the CAP3 contig number. (Accession numbers for ESTs generated in this study will be made available upon acceptance of manuscript). Tag sequence is the 16 bases that were 
sequenced. GATC was added to the $5^{\prime}$ end of these sequences for matching tags to genes. Gene descriptor gives the top BLASTX hit for contigs and newly generated ESTs or the gene descriptor from GenBank. Tag index describes placement of tag along gene, with 0 representing the $3^{\prime}$-most tag and smaller numbers moving progressively toward the 5' end. Position is the base number of the sequence at which the $G$ of GATC begins. Gene length is the number of bases in the sequence and FDR is the False Discovery Rate from the exact test for differential expression between major ampullate glands and cephalothoraxes (see Additional file 2: Table S1).

\section{Abbreviations}

MA: Major ampullate; MPSS: Massively parallel signature sequencing CPM: Counts per million; ORF: Open reading frame; FDR: False discovery rate.

\section{Competing interests}

The authors declare they have no competing interests.

\section{Authors' contributions}

$\mathrm{AKL}$ analyzed the data and drafted the manuscript. CYH helped conceive the experimental design, helped collect data, and revised the manuscript. GBW analyzed the data and revised the manuscript. NAA conceived the experiments, collected data, contributed to analytical design, and helped draft the manuscript. All authors read and approved the final manuscript.

\section{Acknowledgements}

We thank K. Bezold, Washington and Lee University's BIOL 221 Winter 2010 class, and E. Brassfield for their help collecting sequence data. T. Girke and T. Backman contributed tag-processing scripts. J. Satterly assisted in reference formatting. T. Clarke, D. Ireland, J. Satterly, and D. Todd provided helpful comments on the manuscript. This work was supported by the National Science Foundation (IOS-0951886 to NAA, IOS-0951061 to CYH), National Institutes of Health (F32 GM78875-1A to NAA), Army Research Office (W911NF-06-1-0455 to (YH), and Washington and Lee University through Lenfest Summer Fellowships to NAA and GBW.

\section{Author details}

${ }^{1}$ Department of Biology, Washington and Lee University, 204 W. Washington St., Lexington, VA 24450, USA. ${ }^{2}$ Biology Department, University of California Riverside, Riverside, CA 92521, USA.

Received: 16 August 2013 Accepted: 22 November 2013

Published: 2 December 2013

\section{References}

1. Foelix RF: Biology of spiders. 3rd edition. New York: Oxford University Press; 2011.

2. Vollrath F, Knight DP: Liquid crystalline spinning of spider silk. Nature 2001, 410(6828):541-548.

3. Blackledge TA, Hayashi CY: Silken toolkits: biomechanics of silk fibers spun by the orb web spider Argiope argentata (Fabricius 1775). J Exp Biol 2006, 209(13):2452-2461

4. Gosline JM, Guerette PA, Ortlepp CS, Savage KN: The mechanical design of spider silks: from fibroin sequence to mechanical function. J Exp Biol 1999, 202(23):3295-3303.

5. Swanson BO, Blackledge TA, Beltrán J, Hayashi CY: Variation in the material properties of spider dragline silk across species. Appl Phys A Mater Sci Process 2006, 82(2):213-218.

6. Sponner A: Spider silk as a resource for future biotechnologies. Entomological Research 2007, 37(4):238-250.

7. Rising A, Widhe M, Johansson J, Hedhammar M: Spider silk proteins: recent advances in recombinant production, structure-function relationships and biomedical applications. Cell Mol Life Sci 2011, 68(2):169-184.

8. Guerette PA, Ginzinger DG, Weber BHF, Gosline JM: Silk properties determined by gland-specific expression of a spider fibroin gene family. Science 1996, 272(5258):112-115.

9. Gatesy J, Hayashi C, Motriuk D, Woods J, Lewis RV: Extreme diversity, conservation, and convergence of spider silk fibroin sequences. Science 2001, 291(5513):2603-2605.
10. Xu M, Lewis RV: Structure of a protein superfiber: spider dragline silk. Proc Natl Acad Sci USA 1990, 87(18):7120-7124.

11. Hinman MB, Lewis RV: Isolation of a clone encoding a second dragline silk fibroin: Nephila clavipes dragline silk is a two-protein fiber. J Biol Chem 1992, 267(27):19320-19324

12. Garb JE, Hayashi CY: Modular evolution of egg case silk genes across orb-weaving spider superfamilies. Proc Natl Acad Sci USA 2005 102(32):11379-11384.

13. Tian M, Lewis RV: Molecular characterization and evolutionary study of spider tubuliform (eggcase) silk protein. Biochemistry (NY) 2005, 44(22):8006-8012.

14. Zhao A, Zhao T, SiMa Y, Zhang Y, Nakagaki K: Unique molecular architecture of egg case silk protein in a spider: Nephila clavata. J Biochem 2005, 138(5):593-604.

15. Colgin MA, Lewis RV: Spider minor ampullate silk proteins contain new repetitive sequences and highly conserved non-silk-like "spacer regions". Protein Sci 1998, 7(3):667-672.

16. Hayashi CY, Blackledge TA, Lewis RV: Molecular and mechanical characterization of aciniform silk: uniformity of iterated sequence modules in a novel member of the spider silk fibroin gene family. Mol Biol Evol 2004, 21(10):1950-1959.

17. Hayashi CY, Lewis RV: Evidence from flagelliform silk cDNA for the structural basis of elasticity and modular nature of spider silks. J Mol Biol 1998, 275(5):773-784.

18. Blasingame E, Tuton-Blasingame T, Larkin L, Falick AM, Zhao L, Fong J, Vaidyanathan V, Visperas A, Geurts P, Hu X, La Mattina C, Vierra C: Pyriform spidroin 1, a novel member of the silk gene family that anchors dragline silk fibers in attachment discs of the black widow spider, Latrodectus hesperus. J Biol Chem 2009, 284(42):29097-29108.

19. Hayashi $\mathrm{CY}$, Shipley $\mathrm{NH}$, Lewis RV: Hypotheses that correlate the sequence, structure, and mechanical properties of spider silk proteins. Int J Biol Macromol 1999, 24(2-3):271-275.

20. Sponner A, Schlott B, Vollrath F, Unger E, Grosse F, Weisshart K: Characterization of the protein components of Nephila clavipes dragline silk. Biochemistry (N Y) 2005, 44(12):4727-4736.

21. Vasanthavada K, Hu X, Falick AM, La Mattina C, Moore AMF, Jones PR, Yee R, Reza R, Tuton T, Vierra C: Aciniform spidroin, a constituent of egg case sacs and wrapping silk fibers from the black widow spider Latrodectus hesperus. J Biol Chem 2007, 282(48):35088-35097.

22. Ayoub NA, Garb JE, Tinghitella RM, Collin MA, Hayashi CY: Blueprint for a high-performance biomaterial: full-length spider dragline silk genes. PLOS ONE 2007, 2(6):e514

23. Zhao AC, Zhao TF, Nakagaki K, Zhang YS, Sima YH, Miao YG, Shiomi K Kajiura Z, Nagata Y, Takadera M, Nakagaki M: Novel molecular and mechanical properties of egg case silk from wasp spider, Argiope bruennichi. Biochemistry (N Y) 2006, 45(10):3348-3356.

24. Motriuk-Smith D, Smith A, Hayashi CY, Lewis RV: Analysis of the conserved $\mathrm{N}$-terminal domains in major ampullate spider silk proteins. Biomacromolecules 2005, 6(6):3152-3159.

25. Rising A, Hjälm G, Engström W, Johansson J: N-terminal nonrepetitive domain common to dragline, flagelliform, and cylindriform spider silk proteins. Biomacromolecules 2006, 7(11):3120-3124.

26. Garb JE, Ayoub NA, Hayashi CY: Untangling spider silk evolution with spidroin terminal domains. BMC Evol Biol 2010, 10(1):243.

27. Xia XX, Qian ZG, Ki CS, Park YH, Kaplan DL, Lee SY: Native-sized recombinant spider silk protein produced in metabolically engineered Escherichia coli results in a strong fiber. Proc Natl Acad Sci USA 2010, 107 (32):14059-14063.

28. Stark M, Grip S, Rising A, Hedhammar M, Engström W, Hjälm G, Johansson J: Macroscopic fibers self-assembled from recombinant miniature spider silk proteins. Biomacromolecules 2007, 8(5):1695-1701.

29. Gnesa E, Hsia Y, Yarger JL, Weber W, Lin-Cereghino J, Lin-Cerghino G, Tang S, Agari K, Vierra CA: Conserved C-terminal domain of spider tubuliform spidroin 1 contributes to extensibility in synthetic fibers. Biomacromolecules 2012, 13(2):304-312.

30. Spiess K, Ene R, Keenan CD, Senker J, Kremer F, Scheibel T: Impact of initial solvent on thermal stability and mechanical properties of recombinant spider silk films. J Mater Chem 2011, 21(35):13594-13604.

31. Swanson BO, Blackledge TA, Summers AP, Hayashi CY: Spider dragline silk: correlated and mosaic evolution in high-performance biological materials. Evolution 2006, 60(12):2539-2551. 
32. Liu Y, Shao Z, Vollrath F: Elasticity of spider silks. Biomacromolecules 2008, 9(7):1782-1786.

33. Liu Y, Sponner A, Porter D, Vollrath F: Proline and processing of spider silks. Biomacromolecules 2008, 9(1):116-121.

34. Casem ML, Turner D, Houchin K: Protein and amino acid composition of silks from the cob weaver, Latrodectus hesperus (black widow). Int J Biol Macromol 1999, 24(2-3):103-108.

35. Ayoub NA, Hayashi CY: Multiple recombining loci encode MaSp1, the primary constituent of dragline silk, in widow spiders (Latrodectus: Theridiidae). Mol Biol Evol 2008, 25(2):277-286.

36. Gaines WA IV, Marcotte WR Jr: Identification and characterization of multiple Spidroin 1 genes encoding major ampullate silk proteins in Nephila clavipes. Insect Mol Biol 2008, 17(5):465-474

37. Hu X, Kohler K, Falick AM, Moore AMF, Jones PR, Vierra C: Spider egg case core fibers: trimeric complexes assembled from TuSp1, ECP-1, and ECP-2. Biochemistry (N Y) 2006, 45(11):3506-3516.

38. Kohler K, Thayer W, Le T, Sembhi A, Vasanthavada K, Moore AMF, Vierra CA Characterization of a novel class II bHLH transcription factor from the black widow spider, Latrodectus hesperus, with silk-gland restricted patterns of expression. DNA Cell Biol 2005, 24(6):371-380

39. Mardis ER: Next-generation DNA sequencing methods. Annu Rev of Genomics Hum Genet 2008, 9:387-402.

40. Mardis ER: The impact of next-generation sequencing technology on genetics. Trends Genet 2008, 24(3):133-141.

41. Shokralla S, Spall JL, Gibson JF, Hajibabaei M: Next-generation sequencing technologies for environmental DNA research. Mol Ecol 2012, 21(8):1794-1805.

42. Prosdocimi F, Bittencourt D, Da Silva FR, Kirst M, Motta PC, Rech EL: Spinning gland transcriptomics from two main clades of spiders (order: Araneae)-insights on their molecular, anatomical and behavioral evolution. PLOS ONE 2011, 6(6):e21634.

43. Lawrence BA, Vierra CA, Moore AMF: Molecular and mechanical properties of major ampullate silk of the black widow spider, Latrodectus hesperus. Biomacromolecules 2004, 5(3):689-695.

44. Griswold CE, Coddington JA, Hormiga G, Scharff N: Phylogeny of the orb-web building spiders (Araneae, Orbiculariae: Deinopoidea, Araneoidea). Zool J Linn Soc 1998, 123(1):1-99.

45. Moon MJ, Townley MA, Tillinghast EK: Fine structureal analysis of secretory silk production in the black widow spider, Latrodectus mactans. Korean J Biol Sci 1998, 2:145-152.

46. Gregory TR, Shorthouse DP: Genome sizes of spiders. J Hered 2003, 94(4):285-290

47. Ayoub NA, Garb JE, Kuelbs A, Hayashi CY: Ancient properties of spider silks revealed by the complete gene sequence of the prey-wrapping silk protein (AcSp1). Mol Biol Evol 2013, 30(3):589-601.

48. Brenner SE, Levitt M: Expectations from structural genomics. Protein SCi 2000, 9(1):197-200.

49. Brenner SE: Target selection for structural genomics. Nat Struct Biol 2000, 7(SUPPL):967-969.

50. Meyers BC, Tej SS, Vu TH, Haudenschild CD, Agrawal V, Edberg SB, Ghazal H, Decola S: The use of MPSS for whole-genome transcriptional analysis in Arabidopsis. Genome Res 2004, 14(8):1641-1653.

51. Torres TT, Metta M, Ottenwälder B, Schlötterer C: Gene expression profiling by massively parallel sequencing. Genome Res 2008, 18(1):172-177.

52. Zhernakova DV, De Klerk E, Westra H, Mastrokolias A, Amini S, Ariyurek Y, Jansen R, Penninx BW, Hottenga JJ, Willemsen G, De Geus EJ, Boomsma DI, Veldink JH, van den Berg LH, Wijmenga C, Den Dunnen JT, Van Ommen GJ, T Hoen PA, Franke L: DeepSAGE reveals genetic variants associated with alternative polyadenylation and expression of coding and non-coding transcripts. PLOS Genet 2013, 9(6):e1003594.

53. Robinson MD, McCarthy DJ, Smyth GK: edgeR: a bioconductor package for differential expression analysis of digital gene expression data. Bioinformatics 2010, 26(1):139-140.

54. Beiter T, Reich E, Williams RW, Simon P: Antisense transcription: a critical look in both directions. Cell Mol Life Sci 2009, 66(1):94-112.

55. Sun M, Hurst LD, Carmichael GG, Chen J: Evidence for a preferential targeting of 3 '-UTRs by cis-encoded natural antisense transcripts. Nucleic Acids Res 2005, 33(17):5533-5543.

56. Katayama S, Tomaru Y, Kasukawa T, Waki K, Nakanishi M, Nakamura M, Nishida H, Yap CC, Suzuki M, Kawai J, Suzuki H, Carninci P, Hayashizaki Y, Wells C, Frith M, Ravasi T, Pang KC, Hallinan J, Mattick J, Hume DA, Lipovich
L, Batalov S, Engström PG, Mizuno Y, Faghihi MA, Sandelin A, Chalk AM, Mottagui-Tabar S, Liang Z, Lenhard B, Wahlestedt C: Antisense transcription in the mammalian transcriptome. Science 2005, 309(5740):1564-1566.

57. Szostak E, Gebauer F: Translational control by 3'-UTR-binding proteins. Brief Funct Genomics 2013, 12(1):58-65.

58. Blackledge TA, Summers AP, Hayashi CY: Gumfooted lines in black widow cobwebs and the mechanical properties of spider capture silk. Zoology 2005, 108(1):41-46.

59. Blamires SJ, Wu CL, Tso IM: Variation in protein intake induces variation in spider silk expression. PLOS ONE 2012, 7(2):e31626.

60. Casem ML, Collin MA, Ayoub NA, Hayashi CY: Silk gene transcripts in the developing tubuliform glands of the Western black widow, Latrodectus hesperus. J Arachnol 2010, 38(1):99-103.

61. Petersen TN, Brunak S, Von Heijne G, Nielsen H: SignalP 4.0: discriminating signal peptides from transmembrane regions. Nat Methods 2011, 8(10):785-786

62. Kornblihtt AR, Schor IE, Alló M, Dujardin G, Petrillo E, Muñoz MJ: Alternative splicing: a pivotal step between eukaryotic transcription and translation. Nat Rev Mol Cell Biol 2013, 14(3):153-165.

63. Giammartino D, Nishida K, Manley J: Mechanisms and consequences of alternative polyadenylation. Mol Cell 2011, 43:853-866.

64. Bicknell A, Cenik C, Chua H, Roth F, Moore M: Introns in UTRs: why we should stop ignoring them. Bioessays 2012, 34:1025-1034.

65. Wang W, Zinn K, Bjorkman PJ: Expression and structural studies of fasciclin I, an insect cell adhesion molecule. J Biol Chem 1993, 268(January 14):1448-1455.

66. Keebaugh ES, Schlenke TA: Adaptive evolution of a novel Drosophila lectin induced by parasitic wasp attack. Mol Biol Evol 2012, 29(2):565-577.

67. Ao J, Ling E, Yu X: Drosophila C-type lectins enhance cellular encapsulation. Mol Immunol 2007, 44(10):2541-2548.

68. Dicko C, Vollrath F, Kenney JM: Spider silk protein refolding is controlled by changing $\mathrm{pH}$. Biomacromolecules 2004, 5(3):704-710.

69. Pizer L: The pathway and control of serine biosynthesis in Escherichia coli. J Biol Chem 1963, 238(12):3934-3944.

70. Castellano I, Merlino A: Y-Glutamyltranspeptidases: sequence, structure, biochemical properties, and biotechnological applications. Cell Mol Life Sci 2012, 69(20):3381-3394.71.

71. Huang X, Madan A: CAP3: A DNA sequence assembly program. Genome Res 1999, 9(9):868-877.

72. Altschul SF, Gish W, Miller W, Myers EW, Lipman DJ: Basic local alignment search tool. J Mol Biol 1990, 215(3):403-410.

73. Gentleman R, Carey VJ, Bates DM, Bolstad B, Dettling M, Dudoit S, Ellis B, Gautier L, Ge Y, Gentry J, Hornik K, Hothorn T, Huber W, lacus S, Irizarry R, Leisch F, Li C, Maechler M, Rossini AJ, Sawitzki G, Smith C, Smyth G, Tierney $L$, Yang JY, Zhang J: Bioconductor: open software development for computational biology and bioinformatics. Genome Biol 2004, 5:R80

74. Pages H, Aboyoung P, Gentleman R, DebRoy S: Biostrings: string objects representing biological sequences, and matching algorithms: $R$ package version 2.24.1. accessed June 2012

75. Smyth GK: Limma: linear models for microarray data. In Bioinformatics and Computational Biology Solutions using $R$ and Bioconductor. Edited by Gentleman R, Carey VJ, Dudoit S, Irizarry R, Huber W. New York: Springer; 2005:397-420.

76. Robinson MD, Oshlack A: A scaling normalization method for differential expression analysis of RNA-seq data. Genome Biol 2010, 11(3):R25.

77. Robinson MD, Smyth GK: Small-sample estimation of negative binomial dispersion, with applications to SAGE data. Biostatistics 2008, 9(2):321-332.

78. Robinson MD, Smyth GK: Moderated statistical tests for assessing differences in tag abundance. Bioinformatics 2007, 23(21):2881-2887.

79. Benjamini $Y$, Hochberg $Y$ : Controlling the false discovery rate: a practical and powerful approach to multiple testing. J R Stat Soc Series B Stat Methodol 1995, Series B(57):289-300.

doi:10.1186/1471-2164-14-846

Cite this article as: Lane et al:: Complex gene expression in the dragline silk producing glands of the Western black widow (Latrodectus hesperus). BMC Genomics 2013 14:846. 